\title{
Language Impairment Resulting from a de novo Deletion of 7q32.1q33
}

\author{
María S. Jiménez-Romero ${ }^{a, b} \quad$ Montserrat Barcos-Martínez ${ }^{b, c}$ \\ Isabel Espejo-Portero ${ }^{\text {b, c }}$ Antonio Benítez-Burraco ${ }^{d}$ \\ ${ }^{a}$ Department of Psychology, University of Córdoba, ${ }^{\mathrm{b}}$ Maimónides Institute of Biomedical Research, and \\ 'Laboratory of Molecular Genetics, University Hospital 'Reina Sofía', Córdoba, and d Department of Philology, \\ University of Huelva, Huelva, Spain
}

\section{Established Facts}

- Chromosome 7 contains many candidate genes for speech delay and language impairment.

\section{Novel Insights}

- The region 7q32.1q33 emerges as a promising target for our search for new genes related to language dysfunction.

\section{Key Words}

Behavioral disturbances - Copy number variations .

Cognitive delay · Language impairment · 7q32.1q33

\begin{abstract}
We report on a girl who presents with hearing loss, behavioral disturbances (according to the Inventory for Client and Agency Planning) as well as motor and cognitive delay (according to Battelle Developmental Inventories) which have a significant impact on her speech and language abilities [according to the Peabody Picture Vocabulary Test (ed 3), and the Prueba de Lenguaje Oral de Navarra-Revisada (Navarra Oral Language Test, Revised)]. Five copy number variations (CNVs) were identified in the child: arr[hg18] 7q32.1q33(127109685-132492196)×1, 8p23.1(71569007359099) ×1,15q13.1(26215673-26884937)×1,Xp22.33(17245-
\end{abstract}

\section{KARGER}

E-Mail karger@karger.com

www.karger.com/msy
102434) $\times 3$, and Xp22.33(964441-965024) ×3. The pathogenicity of similar CNVs is mostly reported as unknown. The largest deletion is found in a hot spot for cognitive disease and language impairment and contains several genes involved in brain development and function, many of which have been related to developmental disorders encompassing language deficits (dyslexia, speech-sound disorder, and autism). Some of these genes interact with FOXP2. The proband's phenotype may result from a reduced expression of some of these genes.

(c) 2016 S. Karger AG, Basel

Developmental disorders entailing language deficits provide crucial evidence of the molecular and genetic underpinnings of the human faculty of language. In the last decades, many candidate genes for conditions such as 
dyslexia, specific language impairment, or speech-sound disorder have been identified and functionally characterized [reviewed in Benítez-Burraco, 2013]. Rare or sporadic conditions resulting from chromosomal rearrangements or copy number variations (CNVs) provide additional evidence of genetic factors that are important for the development and function of the brain areas involved in language processing. Interestingly, many of them are found in the long arm of chromosome 7 . Specifically, the region $7 \mathrm{q} 31 \mathrm{q} 36$ has been claimed to be a hot spot for the evolution of human-specific communication abilities [Schneider et al., 2012]. The renowned 'language gene' FOXP2 maps within this region (7q31). FOXP2 is crucial for the development of brain circuits involved in procedural learning and is found mutated in people with speech and language problems [Fisher and Scharff, 2009; Schreiweis et al., 2014]. The region has also been related to disorders involving phonological deficits, such as dyslexia or speech-sound disorder [Kaminen et al., 2003; Peter et al., 2012] but also to autism [Beyer et al., 2001; Bonora et al., 2002]. In this study, we report on a girl presenting with language and cognitive delay, motor problems, hearing loss, and behavioral disturbances, who bears 5 de novo CNVs in chromosomes 7, 8, 15, and X. The largest deletion is located within the region $7 \mathrm{q} 32.1 \mathrm{q} 33$. Given the phenotype of this patient, we hypothesize that some gene(s) important for language development may have been affected by this deletion.

\section{Patient and Methods}

\section{Case Presentation}

The proband was born after 41 weeks of gestation to a 28 -yearold female. The proband's parents were healthy and nonconsanguineous. During pregnancy, intrauterine growth restriction was reported. At birth, the girl's weight was 2,545 $\mathrm{g}$ (3rd percentile) her height $50 \mathrm{~cm}$ (62nd percentile) and her head circumference was $33 \mathrm{~cm}$ (18th percentile). APGAR evaluation scores were normal (8/9). Cord blood pH was 7.30. Dystocia and fetal distress were reported at delivery. Because of skin pallor and mild respiratory distress at birth, the newborn was kept in an incubator for 8 days. Further exploration was indicative of hypotonia, sucking weakness, and absence of crying (until 3 years of age). Ophthalmologic examination and brain MRI, performed at 3 years, yielded normal results, although mild microcephaly was reported. The child has shown no signs of epilepsy. At 3.6 years, she was diagnosed with severe bilateral sensorineural hearing loss (neuropathy). Because her medical history was significant for numerous episodes of serous otitis media, ear drainage tubes were placed. From age 4 until present, she has used behind-the-ear hearing aids. An audiometry test (auditory steady-state response) performed at 7 years revealed hearing loss in both ears (right ear: objective threshold of $47 \mathrm{~dB}$ correlated with less than $10 \mathrm{~dB}$ on subjective thresholds; left ear: objective threshold of $72 \mathrm{~dB}$ correlated with less than $10 \mathrm{~dB}$ on subjective thresholds). Mouth, tongue, teeth, and palate examination performed at 7.7 years revealed no abnormalities.

\section{Cognitive, Linguistic, and Behavioral Evaluation}

The patient's global development was assessed with the Spanish version of the Battelle Developmental Inventories [De la Cruz López and González Criado, 2011]. This test comprises 341 items and includes specific subtests for evaluating receptive and expressive communication skills, gross and fine motor skills, cognitive development, personal/social development, and adaptive abilities. Language problems were assessed in detail with the Spanish version of the Peabody Picture Vocabulary Test, edition 3 (PPVT-3) [Dunn et al., 2006] and the Prueba de Lenguaje Oral de Navarra-Revisada (PLON-R; Navarra Oral Language Test, Revised) [Aguinaga et al., 2004]. PPVT-3 is a receptive vocabulary test that assesses the correct acquisition of words by the child. The child is asked to point to 1 of 4 color pictures on a page after hearing a word named by the examiner. PLON-R is an expressive language test which evaluates phonological, morphological, semantic, and syntactic aspects of the child discourse but also how the child puts acquired language knowledge into practice (including how language use is planned, auto-regulated, and modified according to the communicative environment). Behavioral problems were assessed with the Spanish version of the Inventory for Client and Agency Planning (ICAP) [Montero, 1996]. This test measures the frequency and severity of 8 types of behavioral disturbances, which are organized in 3 subscales: asocial maladaptive behavior (uncooperative behavior and socially offensive behavior), internalized maladaptive behavior (withdrawn or inattentive behavior, unusual or repetitive habits, and self-harm), and externalized maladaptive behavior (disruptive behavior, destructive to property, and hurtful to others). Problem behaviors are scored as present or absent. As a result, behavior is rated as normal or abnormal, with behavioral problems rated as marginally serious, moderately serious, serious, or very serious.

\section{Molecular Cytogenetic Analysis}

Karyotype Analysis

Peripheral venous blood lymphocytes were grown following standard protocols and collected after $72 \mathrm{~h}$. A moderate resolution G-banding (550 bands) using trypsin (Gibco ${ }^{\circledR} 1 \times$ trypsin and Leishmann stain) was performed. Microscopic analysis was conducted with a Nikon ${ }^{\circledR}$ eclipse 50i optical microscope and the Ikaros karyotyping system (MetaSystem ${ }^{\circledR}$ software).

\section{Fragile X Syndrome Determinants}

CGG expansions affecting the FMR1 gene (the main determinant for fragile $\mathrm{X}$ syndrome) were analyzed according to standard protocols. Polymerase chain reaction (PCR) of the fragile site was performed with specific primers and the resulting trinucleotide repeat fragments were differentiated and evaluated by agarose electrophoresis.

\section{Angelman Syndrome Determinants}

FISH with the LSI Prader-Willi/Angelman probes (15q11q13 SNPRN and 15q11q13 D15S10 Izasa $^{\circledR}$ ) was conducted to detect microdeletions in the $15 \mathrm{q} 11 \mathrm{q} 13$ locus and/or in the UBE3A gene, located at 15q11.2. Metaphase spreads were harvested from peripheral blood as mentioned above. Slides were analyzed with a Nikon eclipse 50i optical microscope and the MetaSystem Isis software. 
Fig. 1. The proband's developmental profile according to the Battelle Developmental Inventories. The obtained scores are shown as relative values referred to the expected scores according to the chronological age of the child. CA = Chronological age; $\mathrm{DA}=$ developmental age.

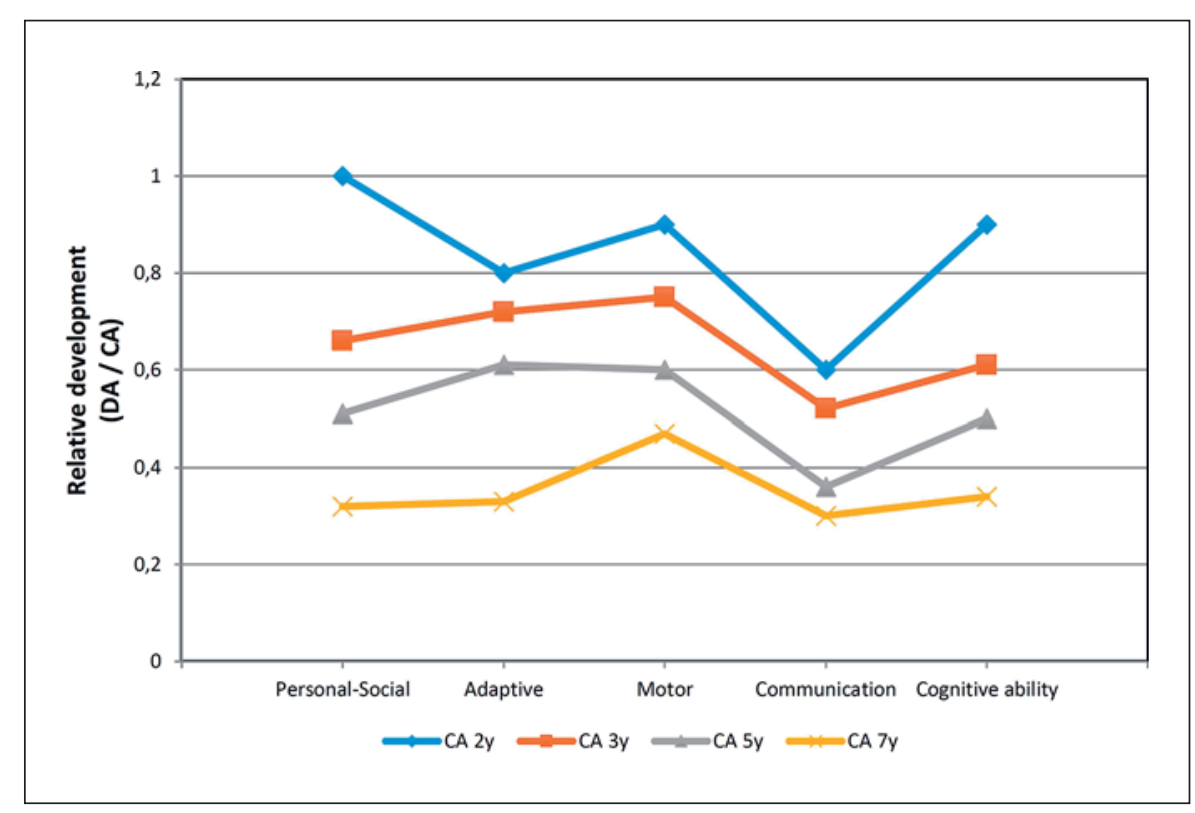

Microarrays for CNV Search and Chromosome Aberration Analysis

DNA samples (total amount: $500 \mathrm{ng}$, concentration: $51.2 \mathrm{ng} / \mu \mathrm{l}$ in EDTA) extracted from blood were hybridized and analyzed on a CGH platform (CytoChip Oligo ISCA 60K), using a DLRS value of 0.15 . The platform included 60.000 probes. Data were analyzed by ADM-2 (Agilent Technologies, Santa Clara, Calif., USA) (threshold $=6.0$; aberrant regions had more than 5 consecutive probes).

\section{Results}

\section{Language and Cognitive Development}

Early developmental milestones were achieved normally by the child. The girl was able to sit without aid at 8 months and walked at 1.6 years. Nonetheless, at the age of 2 years, her parents reported an absence of speech and lack of interest in her social environment. In order to accurately evaluate her global development, the Spanish version of the Battelle Developmental Inventories was administered at ages 2,3,5, and 7. The obtained scores pointed to a broad developmental delay which has exacerbated over the years and has an impact mostly on language abilities. Accordingly, communication skills were severely impaired as well as cognitive and personal-social abilities, and adaptive abilities and motor skills were relatively spared (fig. 1). From age 3 onwards, the proband has exhibited an impulsive behavior. At 7.7 years, the Spanish version of ICAP was administered in order to evaluate her behavioral disturbances. The obtained scores were indicative of moderately-serious asocial maladaptive behavior (uncooperative behavior) and of moderately-serious externalized maladaptive behavior (disruptive behavior and hurtful to others). The ICAP scores were in line with the scores obtained in the Battelle subtest for personal-social abilities, which were suggestive of problems for correctly interacting with her peers and for establishing emotional links, and ultimately, of uncooperative and disruptive behavior. Because of the difficulties experienced by the proband, she has attended a special education unit since the age of 6 . From 6 years until present, she has been learning Spanish sign language, which was thought to aid in improving her communication abilities.

At 7.7 years, her language problems were assessed in detail. Language comprehension abilities were assessed with the Spanish version of PPVT-3, whereas language production was assessed with PLON-R and by a natural speech sample. In PPVT-3, the proband scored 47 points (3.9 years below expected). In PLON-R, she scored 4 years below her typically developing peers. In her discourse, she exhibited phonemic paraphasias that are usually found in younger children, including reductions of consonant clusters, deletions of unstressed syllables, assimilations, and substitutions. Nonetheless, the child was able to properly articulate all the Spanish phonemes separately. On the morphological and syntactic side, she had problems correctly repeating the target sentences 


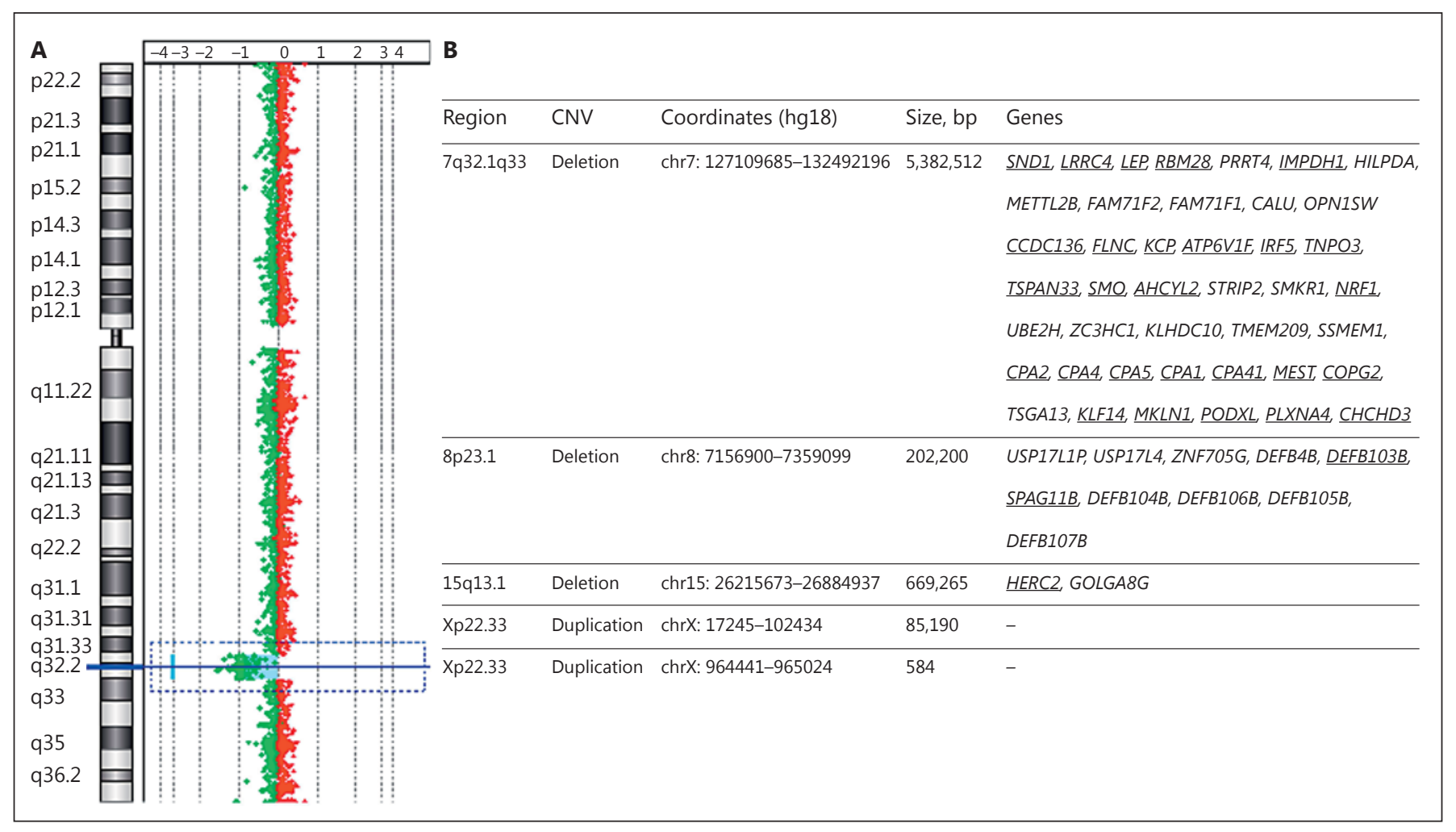

Fig. 2. Array CGH and deleted genes in the proband. A Array CGH of the proband's chromosome 7 showing the microdeletion in 7q32.1q33. B Summary table of the genes deleted in the proband. Only genes encoding proteins are displayed. OMIM genes are underlined.

provided by the experimenter. Hence, in the elicited production tasks of PLON-R, the proband usually employed simpler syntactic structures (list of words mostly). Similarly, she correctly generated terms for colors, spatial relations, body parts, and simple actions but only when the test for children aged 3 was used. Overall, the semantics of the proband's sentences was acceptable, but the syntax was largely agrammatical. The analysis of spontaneous speech samples generated by the child supported the view that her language was structurally simpler than that of her typically developing peers. Accordingly, she only produced simple enunciative and interrogative sentences about her surrounding environment or favorite topics. Verbs and functional words were usually omitted. The sentence modality was correctly marked by prosodic cues (i.e., voice inflections). Linguistically, the child's impulsive behavior and lack of self-control resulted in logorrhea (usually, repetitive fragments of speech), but no signs of echolalia were observed. Interestingly, the child used to 'sing without words' privately (i.e., she generated an unstructured melody) as a sort of self-stimula- tion whenever she was compelled to fulfill tasks that were difficult to achieve (e.g., those encompassing the evaluation tests). Her language abilities with Spanish sign language were also evaluated, although they did not differ from her abilities in the oral domain. Overall, comprehension clearly surpassed production and signs were poorly articulated.

\section{Molecular Cytogenetic Analysis}

Routine molecular cytogenetic analyses were performed at the age of 3.5 years. PCR analysis of the FMR1 fragile site resulted normal. FISH analyses of the loci $S N R P N$ and $U B E 3 A$ were also normal. No major chromosomal rearrangements were observed in the karyotype analysis. Because the analysis did not discard the presence of low-frequency mosaicisms and/or cryptic chromosomal alterations, an array CGH was performed. The microarray identified 5 CNVs: one large deletion within the region 7q32.1q33 (chr7: 127109685-132492196, hg18), 2 smaller deletions within regions 8p23.1 (chr8: 7156900-7359099, hg18) and 15q13.1 (chr15: 26215673- 
26884937, hg18), and 2 microduplications within region Xp22.33 (chrX: 17245-102434, hg18, and chrX: 964441965024, hg18) (fig. 2). The parents' karyotypes were normal and the microarrays did not detect any CNVs.

According to the DECIPHER (www.decipher.sanger. ac.uk) database, the pathogenicity of these CNVs is largely unknown. The fragment deleted in chromosome 8 contains 14 genes, of which 10 encode proteins (fig. 2). The smallest microdeletion encompassing the whole region lost in our proband was characterized as likely benign in a previous case with moderate global developmental delay (DECIPHER patient 285572). The loss of an overlapping region is reported as of unknown pathogenicity (patient 323189). Regarding the CNV loss in chromosome 15 , the deleted fragment contains 15 genes, of which only 2 encode proteins (fig. 2). The smallest deletion encompassing the whole region deleted in our proband was classified as definitely pathogenic in a patient featuring abnormality of skull, autism, and intellectual disability (patient 327123), although this deletion is 6-fold larger. Patients bearing CNVs which overlap with the distal part of the region deleted in our proband $(285591,273128)$ showed delayed speech and language development. These CNVs are reported as of unknown or uncertain pathogenicity, although they do not contain any of the genes deleted in our proband. The regions duplicated in chromosome X contain no genes (fig. 2). Similar deletions are found in patients with complex rearrangements only. Finally, the region deleted in chromosome 7 contains 70 genes, of which 42 encode proteins (fig. 2). Smaller CNV losses within this region are classified as of unknown pathogenicity. One patient (280769) was reported to suffer from cognitive impairment, speech and language delay, and motor delay. CNV losses of overlapping regions are also mostly reported as of unknown pathogenicity. Patients bearing these CNV losses are intellectually disabled (1297, 265853, and 271939), and in some cases, they also exhibit delayed speech and language development (patient 1296).

\section{Discussion}

We have evaluated in detail the phenotypic profile of a child with severe language impairment (particularly in the oral domain), cognitive and behavioral disturbances, and with several de novo CNVs in chromosomes 7, 8, 15, and X. Similar CNVs are reported as of unknown pathogenicity. Several candidate genes for language and cognitive function are found in the deleted regions.
With regards to the language deficits exhibited by our proband, the region deleted in $7 \mathrm{q} 32.1 \mathrm{q} 33$ contains several targets of FOXP2, including CALU, IMPDH1, LEP, MEST, OPN1SW, and RBM28 [Vernes et al., 2007, 2011]. FOXP2 is a well-known language-related gene that contributes to the development of cortico-thalamic-striatal circuits involved in procedural learning; mutations in this gene are associated with speech and language problems [Fisher and Scharff, 2009; Schreiweis et al., 2014]. Interestingly, CALU encodes calumenin, a calcium-binding protein of the endoplasmic reticulum. This gene is highly expressed during early development in different brain regions, particularly in migrating neurons [Vasiljevic et al., 2012]. Mutations in RBM 28 affect ribosome biogenesis and the structure of the rough endoplasmic reticulum, and give rise to microcephaly, moderate to severe mental retardation, and progressive motor deterioration, resulting from upper and lower motor dysfunction [Nousbeck et al., 2008].

Concerning the proband's developmental delay, 2 genes of interest are also located in the fragment deleted in chromosome 7: PODXL and NRF1. Both of them play a role in brain development and function. The former encodes a polysialylated neural adhesion protein involved in neurite growth, neuron branching, and axonal fasciculation; in mice, the loss of Podxl function results in a reduced number of synapses in the central nervous system and in the neuromuscular system [Vitureira et al., 2010]. $N R F 1$ encodes a transcription factor that regulates many nuclear genes that are essential for mitochondrial function; this gene seems to be relevant to the pathogenesis of neurodegenerative diseases via perturbation of mitochondrial and extra-mitochondrial functions [Satoh et al., 2013]. Also of interest, regarding the probands' cognitive profile, is the HERC2 gene located in the deleted region in chromosome 15. According to Puffenberger et al. [2012], mutations in HERC2 affect E3 ubiquitin ligase activity, resembling the pathophysiologic mechanism underlying Angelman syndrome. This gene is a candidate gene for autosomal recessive mental retardation-38, a condition involving motor, speech, adaptive, and social delay, autistic features, aggression, impulsivity, and distractibility [Puffenberger et al., 2012]. Finally, it is worth noting that the deleted fragment in chromosome 7 contains 2 targets of RUNX2 (CPA4 and MKLN1). RUNX2 encodes an osteogenic factor that controls the closure of cranial sutures and several aspects of brain growth, and it has been related to most of the changes that brought about our more globular brain(case) and our speciesspecific mode of cognition, including language [Boeckx 
and Benítez-Burraco, 2014; Benítez-Burraco and Boeckx, 2015].

Regarding the behavioral problems exhibited by our proband, it is worth noting that several of the deleted genes in chromosome 7 belong to the autism susceptibility locus in 7q32, including both imprinted genes (MEST, CPA4, CPA5, and KLF14) and nonimprinted genes (COPG2 and TSGA14). Interestingly, COPG2 expression is downregulated in the human cortex compared to chimpanzees, supporting a role for this gene in the development of a 'social brain' [according to Schneider et al., 2012]. Similarly, point mutations in TSGA14 [Korvatska et al., 2011] and UBE2H [Vourc'h et al., 2003] have been identified in families with autism spectrum disorders. The latter encodes an E2 (ubiquitin-conjugating) enzyme involved in neural development (intriguingly, another member of this system, UBE3A, causes Angelman syndrome). Changes in the expression levels of imprinted genes within this region have been related to Russell-Silver syndrome as well [Peñaherrera et al., 2010], a condition involving intrauterine growth retardation, poor postnatal growth, and craniofacial malformations [Price et al., 1999].

Concerning the motor problems exhibited by the child, we wish to highlight the filamin $\mathrm{C}$ gene (FLNC) associated to myofibrillar myopathy [Tasca et al., 2012].

Finally, with regards to her hearing problems, we find of interest that both $S M O$ and $U B E 2 H$ are expressed in the cochlea [Friedman and Avraham, 2009]. Also, most of the genes in the deleted region in chromosome 8 encode defensins, some of which (specifically DEFB4) have been linked to an enhanced susceptibility to infectious diseases affecting the ear (particularly, otitis media) [Jones et al., 2014].

In conclusion, although the exact genetic cause of the language and cognitive delay exhibited by our proband remains to be fully elucidated, we believe that the hemizygosity of the genes discussed above may explain most of her clinical problems. We expect that this case (and the genes we highlight in this study) contributes to a better understanding of the genetic underpinnings of the human faculty of language.

\section{Acknowledgments}

We would like to thank the proband and her family for their participation in this study. We wish to thank Wanda Lattanzi for reading the final draft of the manuscript and for her assistance with the liftOver tool of the UCSC Genome Browser. This work was supported by the Spanish Ministry of Economy and Competitiveness (grant FFI2014-61888-EXP to A.B.-B.).

\section{Statement of Ethics}

Ethics approval for this research was granted by the Comite Ético del Hospital Universitario 'Reina Sofía'. Written informed consent was obtained from the proband's parents for publication of this case report and of any accompanying tables and images.

\section{Disclosure Statement}

The authors declare no conflicts of interest.

\section{References}

Aguinaga G, Armentia M, Fraile A, Olangua P, Bonora E, Bacchelli E, Levy ER, Blasi F, Marlow Uriz N: PLON-R Prueba de Lenguaje Oral de Navarra (TEA Ediciones, Madrid 2004).

Benítez-Burraco A: Genetics of language: roots of specific language deficits, in Boeckx C, Grohmann KK (eds): The Cambridge Handbook of Biolinguistics, pp 375-412 (Cambridge University Press, Cambridge 2013).

Benítez-Burraco A, Boeckx C: Possible functional links among brain- and skull-related genes selected in modern humans. Front Psychol 6: 794 (2015)

Beyer KS, Klauck SM, Wiemann S, Poustka A: Construction of a physical map of an autism susceptibility region in 7q32.3-q33. Gene 272: 85-91 (2001).

Boeckx C, Benítez-Burraco A: The shape of the human language-ready brain. Front Psychol 5:282 (2014)
A, et al: Mutation screening and imprinting analysis of four candidate genes for autism in the 7q32 region. Mol Psychiatry 7:289-301 (2002).

De La Cruz López MV, González Criado M Adaptación española del inventario de Desarrollo Battelle (TEA Ediciones, Madrid 2011).

Dunn L, Dunn LM, Arribas D: Peabody, test de vocabulario en imágenes (TEA Ediciones, Madrid 2006).

Fisher SE, Scharff C: FOXP2 as a molecular window into speech and language. Trends Genet 25:166-177 (2009).

Friedman LM, Avraham KB: MicroRNAs and epigenetic regulation in the mammalian inner ear: implications for deafness. Mamm Genome 20:581-603 (2009).
Jones EA, Kananurak A, Bevins CL, Hollox EJ, Bakaletz LO: Copy number variation of the beta defensin gene cluster on chromosome $8 \mathrm{p}$ influences the bacterial microbiota within the nasopharynx of otitis-prone children. PLoS One 9:e98269 (2014).

Kaminen N, Hannula-Jouppi K, Kestilä M, Lahermo P, Muller K, et al: A genome scan for developmental dyslexia confirms linkage to chromosome 2p11 and suggests a new locus on 7q32. J Med Genet 40:340-345 (2003).

-Korvatska O, Estes A, Munson J, Dawson G, Bekris LM, et al: Mutations in the TSGA14 gene in families with autism spectrum disorders. Am J Med Genet B Neuropsychiatr Genet 156B:303-311 (2011).

Montero D: Evaluación de la conducta adaptativa en personas con discapacidades. Adaptación y validación del ICAP (Mensajero Ediciones, Bilbao 1996) 
Nousbeck J, Spiegel R, Ishida-Yamamoto A, Indelman M, Shani-Adir A, et al: Alopecia, neurological defects, and endocrinopathy syndrome caused by decreased expression of RBM28, a nucleolar protein associated with ribosome biogenesis. Am J Hum Genet 82: 1114-1121 (2008).

Peñaherrera MS, Weindler S, Van Allen MI, Yong SL, Metzger DL, et al: Methylation profiling in individuals with Russell-Silver syndrome. Am J Med Genet A 152A:347-355 (2010).

Peter B, Matsushita M, Raskind WH: Motor sequencing deficit as an endophenotype of speech sound disorder: a genome-wide linkage analysis in a multigenerational family. Psychiatr Genet 22:226-234 (2012)

- Price SM, Stanhope R, Garrett C, Preece MA, Trembath RC: The spectrum of Silver-Russell syndrome: a clinical and molecular genetic study and new diagnostic criteria. J Med Genet 36:837-842 (1999).

- Puffenberger EG, Jinks RN, Wang H, Xin B, Fiorentini $\mathrm{C}$, et al: A homozygous missense mutation in HERC2 associated with global developmental delay and autism spectrum disorder. Hum Mutat 33:1639-1646 (2012).
Satoh J, Kawana N, Yamamoto Y: Pathway analysis of ChIP-Seq-based NRF1 target genes suggests a logical hypothesis of their involvement in the pathogenesis of neurodegenerative diseases. Gene Regul Syst Bio 7:139-152 (2013).

- Schneider E, Jensen LR, Farcas R, Kondova I, Bontrop RE, et al: A high density of human communication-associated genes in chromosome 7q31-q36: differential expression in human and non-human primate cortices. Cytogenet Genome Res 136:97-106 (2012).

Schreiweis C, Bornschein U, Burguière E, Kerimoglu C, Schreiter S, et al: Humanized Foxp2 accelerates learning by enhancing transitions from declarative to procedural performance. Proc Natl Acad Sci USA 111:14253-14258 (2014).

- Tasca G, Odgerel Z, Monforte M, Aurino S, Clarke NF, et al: Novel FLNC mutation in a patient with myofibrillar myopathy in combination with late-onset cerebellar ataxia. Muscle Nerve 46:275-282 (2012).
Vasiljevic M, Heisler FF, Hausrat TJ, Fehr S, Milenkovic I, et al: Spatio-temporal expression analysis of the calcium-binding protein calumenin in the rodent brain. Neuroscience 202:29-41 (2012).

-Vernes SC, Spiteri E, Nicod J, Groszer M, Taylor JM, et al: High-throughput analysis of promoter occupancy reveals direct neural targets of FOXP2, a gene mutated in speech and language disorders. Am J Hum Genet 81:12321250 (2007).

-Vernes SC, Oliver PL, Spiteri E, Lockstone HE, Puliyadi R, et al: Foxp2 regulates gene networks implicated in neurite outgrowth in the developing brain. PLoS Genet 7:e1002145 (2011).

Vitureira N, Andrés R, Pérez-Martínez E, Martínez A, Bribián A, et al: Podocalyxin is a novel polysialylated neural adhesion protein with multiple roles in neural development and synapse formation. PLoS One 5:e12003 (2010).

Vourc'h P, Martin I, Bonnet-Brilhault F, Marouillat S, Barthélémy C, et al: Mutation screening and association study of the $U B E 2 H$ gene on chromosome $7 \mathrm{q} 32$ in autistic disorder. Psychiatr Genet 13:221-225 (2003). 